doi:10.13108/2021-13-3-17

\title{
DIRICHLET BOUNDARY VALUE PROBLEM FOR EQUATIONS DESCRIBING FLOWS OF A NONLINEAR VISCOELASTIC FLUID IN A BOUNDED DOMAIN
}

\author{
M.A. ARTEMOV, YU.N. BABKINA
}

\begin{abstract}
We consider a boundary value problem for a mathematical model describing a stationary isotermic flow of a nonlinear viscoelastic liquid with a varying viscosity depending on the shear rate in a bounded three- or two-dimensional domain with a sufficiently smooth boundary. We assume that the viscosity function is continuous and bounded. The considered model is a system of strongly nonlinear third order partial differential equations. The boundary of the flow region is subject to the homogeneous Dirichlet boundary condition, which corresponds to the standard condition of adhesion on the solid walls of a vessel. This boundary value problem is considered in a weak (generalized) sense. A weak solution is a pair of functions "velocity-pressure" satisfying the equations of motion in the distribution sense. Using the regularization method via introducing terms with an additional viscosity into the equations, we construct a family of auxiliary approximating problems. We provide an interpretation of the problems of this family in the form of operator equation with a continuous nonlinearoperator satisfying the $\alpha$-monotonicity condition. On the base of a solvability theorem for equations with $\alpha$-operators, we prove the existence of at least one solution for each positive value of the additional viscosity. We obtain estimates for the norms of solutions independent of the additional viscosity parameter. The solution to the original boundary value problem is obtained as the limit of the sequence of solutions to approximating problems as the additional viscosity tends to zero. The passage to the limit is carried out on the base of well-known results on the compactness of the embedding of Sobolev spaces and Lebesgue theorem on dominated convergence. In addition, we establish an energy-type estimate for the vector velocity function.
\end{abstract}

Keywords: Dirichlet boundary value problem, existence theorem, weak solution, $\alpha$ operator, regularization method, additional viscosity, nonlinear viscoelastic fluid, polymer solution.

Mathematics Subject Classification: 35Q35, 35A01

\section{INTRODUCTION}

It is commonly believed that the following system of equations describes well isothermal flows of low-concentrated water solutions of polymers [1]:

$$
\begin{gathered}
\frac{\partial \boldsymbol{v}}{\partial t}+\sum_{i=1}^{n} v_{i} \frac{\partial \boldsymbol{v}}{\partial x_{i}}-\operatorname{div}_{\mathbf{x}}\left[\nu \mathcal{E}(\boldsymbol{v})+\kappa \frac{\partial \mathcal{E}(\boldsymbol{v})}{\partial t}+\kappa \sum_{i=1}^{n} v_{i} \frac{\partial \mathcal{E}(\boldsymbol{v})}{\partial x_{i}}\right]+\nabla_{\mathbf{x}} \pi=\boldsymbol{f} \\
\operatorname{div}_{\mathbf{x}} \boldsymbol{v}:=\sum_{i=1}^{n} \frac{\partial v_{i}}{\partial x_{i}}=0
\end{gathered}
$$

M.A. Artemov, Yu.N. Babkina, Dirichlet boundary value problem for equations Describing FLOWS OF A NONLINEAR VISCOELASTIC FLUID IN A BOUNDED DOMAIN.

(C) Artemov M.A., Babkina Yu.N. 2021.

Submitted July 19, 2020. 
where $n$ is the dimension of the space, $n=2,3 ; x_{1}, \ldots, x_{n}$ are spatial coordinates; $t$ is the time; $\boldsymbol{v}$ is a shear rate; $\pi$ is a pressure; $\boldsymbol{f}$ is a density of external forces; $\mathcal{E}(\boldsymbol{v})=\left(\mathcal{E}_{i, j}(\boldsymbol{v})\right)$ is a strain velocity tensor, $\mathcal{E}_{i, j}(\boldsymbol{v})=\frac{1}{2}\left(\frac{\partial v_{i}}{\partial x_{j}}+\frac{\partial v_{j}}{\partial x_{i}}\right) ; \nu$ is a kinematic viscousity coefficient, $\nu>0 ; \kappa$ is a viscosity relaxation coefficient, $\kappa>0$.

The first results on solvability of the motion model of an incompressible viscous medium with polymer additives were obtained by A.P. Oskolkov [2]-4] under the condition of adhesion at the boundary of the flow region. The case of a weakly compressible polymer fluid was investigated by G.A. Sviridyuk [5]. The case when the flow domain can be unbounded was considered in [6]. The existence and uniqueness of a $k$-weak solution to the initial-boundary value problem for model (1.1), (1.2) under the Navier slip boundary condition were studied in [7]. Existence theorems for other types of inhomogeneous boundary value problems were also proved [8]-[11] for various simplified versions of the motion equations (1.1). The boundary value problem describing heat transfer in a flow of polymer solution was studied in [12].

In the present paper we consider a generalization of the model of water polymer solutions. In contrast to the above works, the viscosity $\nu$ is assumed to be not a constant, but depending on the shear rate: $\nu=\nu(|\mathcal{P} \mathcal{E}(\boldsymbol{v})|)$, where $\mathcal{P}$ is some operator. If $\mathcal{P}$ coincides with the identity mapping, then we obtain a classical nonlinear viscoelastic liquid [13]. In the case when $\mathcal{P}$ is an averaging operator, we get a model of nonlocal type [14].

We dwell on the case of a stationary flow, that is, as the quantities $\boldsymbol{v}, \pi$ and $\boldsymbol{f}$ are independent of the time $t$ and we assume that the liquid flows inside a bounded domain $\Omega \subset \mathbb{R}^{n}$. Then we arrive at the following system of equations in $\Omega$ :

$$
\sum_{i=1}^{n} v_{i} \frac{\partial \boldsymbol{v}}{\partial x_{i}}-\operatorname{div}_{\mathbf{x}}\left[\nu(|\mathcal{P} \mathcal{E}(\boldsymbol{v})|) \mathcal{E}(\boldsymbol{v})+\kappa \sum_{i=1}^{n} v_{i} \frac{\partial \mathcal{E}(\boldsymbol{v})}{\partial x_{i}}\right]+\nabla_{\mathbf{x}} \pi=\boldsymbol{f}, \quad \operatorname{div}_{\mathbf{x}} \boldsymbol{v}=0
$$

On the boundary of $\Omega$, the Dirichlet condition is imposed and this corresponds to the standard adhesion condition on solid walls of a vessel:

$$
\boldsymbol{v}=\mathbf{0} \quad \text { on } \quad \partial \Omega
$$

The main aim of this work is to find conditions ensuring the solvability of boundary value problem (1.3), (1.4). With the help of the regularization method based on introducing "vanishing viscosity" [4], the theorem on the solvability of equations with $\alpha$-operators [15] and the passage to the limit, we prove that this problem has at least one weak solution $(\boldsymbol{v}, \pi)$ in the Cartesian product $\mathbf{H}^{1}(\Omega) \times \mathfrak{D}^{\prime}(\Omega)$ under the assumption that the viscosity function is continuous and bounded, the operator $\mathcal{P} \mathcal{E}$ is strongly continuous, and the boundary of the domain $\Omega$ has the smoothness $\mathcal{C}^{3}$.

We mention that in work [16], on base of Cosserat theory, there was studied a one-dimensional version of a model of a non-stationary flow of a nonlinear viscoelastic liquid of this type.

\section{Weak SOlutions of BOUNDARY VAlue PROBlem (1.3), 1.4 AND MAIN RESULT OF WORK}

We begin with introducing notations, operators and functional spaces.

As usually, $\mathbb{N}:=\{1,2, \ldots\}, \mathbb{R}:=(-\infty,+\infty)$ and $\mathbb{R}_{+}:=[0,+\infty)$.

By $\boldsymbol{X}: \boldsymbol{Y}$ we denote the scalar product of real $n \times n$-matrices $\boldsymbol{X}=\left(X_{i, j}\right)$ and $\boldsymbol{Y}=\left(Y_{i, j}\right)$, that is,

$$
\boldsymbol{X}: \boldsymbol{Y}:=\sum_{i, j=1}^{n} X_{i, j} Y_{i, j}
$$


Depending on the context, the symbol $|\cdot|$ denotes either an absolute value of a real number or the Euclidean norm of a vector or matrix; in particular,

$$
|\boldsymbol{X}|:=(\boldsymbol{X}: \boldsymbol{X})^{1 / 2} \text {. }
$$

We define differential operators:

$$
\begin{gathered}
\nabla_{\mathbf{x}}:=\left(\frac{\partial}{\partial x_{1}}, \cdots, \frac{\partial}{\partial x_{n}}\right), \quad \nabla_{\mathbf{x}}^{2}:=\nabla_{\mathbf{x}} \cdot \nabla_{\mathbf{x}}=\Delta_{\mathbf{x}}=\sum_{i=1}^{n} \frac{\partial^{2}}{\partial x_{i}^{2}} \\
\nabla_{\mathbf{x}}^{3}:=\nabla_{\mathbf{x}}\left(\nabla_{\mathbf{x}}^{2}\right)=\left(\frac{\partial}{\partial x_{1}} \nabla_{\mathbf{x}}^{2}, \ldots, \frac{\partial}{\partial x_{n}} \nabla_{\mathbf{x}}^{2}\right), \quad \ldots, \quad \nabla_{\mathbf{x}}^{m}:=\nabla_{\mathbf{x}}\left(\nabla_{\mathbf{x}}^{m-1}\right) .
\end{gathered}
$$

Let

$$
\mathfrak{D}(\Omega):=\left\{\phi: \Omega \rightarrow \mathbb{R} \mid \phi \in C^{\infty}(\Omega), \operatorname{supp} \phi \subset \Omega\right\} .
$$

By $\mathfrak{D}^{\prime}(\Omega)$ we denote the space of distributions on $\Omega$.

We shall employ usual notations for the Lebesgue spaces $L_{p}(\Omega), p \in[1, \infty)$, and for Sobolev spaces $H^{m}(\Omega), H_{0}^{m}(\Omega), m \in \mathbb{N}$, of functions defined on the domain $\Omega$. The norms in these spaces are defined in a standard way [17]. The spaces of vector functions are always denoted by a bold font: $\mathbf{L}_{p}(\Omega), \mathbf{H}^{m}(\Omega)$, etc. The dual space for $H_{0}^{m}(\Omega)$ is denoted by $H^{-1}(\Omega)$.

Let

$$
\mathcal{V}(\Omega):=\left\{\phi: \Omega \rightarrow \mathbb{R}^{n} \mid \boldsymbol{\phi} \in \boldsymbol{C}^{\infty}(\Omega), \operatorname{supp} \phi \subset \Omega, \operatorname{div}_{\mathbf{x}} \phi=0\right\}
$$

The closure of the set of vector functions $\mathcal{V}(\Omega)$ in the Sobolev spaces $\mathbf{H}^{1}(\Omega), \mathbf{H}^{2}(\Omega)$ and $\mathbf{H}^{3}(\Omega)$ gives three main spaces which will be employed in studying boundary value problem (1.3), (1.4); we denote them respectively by $\mathbf{V}_{1}(\Omega), \mathbf{V}_{2}(\Omega)$ and $\mathbf{V}_{3}(\Omega)$.

In the space $\mathbf{V}_{1}(\Omega)$ we introduce a scalar product and a norm:

$$
(\boldsymbol{v}, \boldsymbol{w})_{\mathrm{V}_{1}(\Omega)}:=\int_{\Omega} \mathcal{E}(\boldsymbol{v}): \mathcal{E}(\boldsymbol{w}) \boldsymbol{d} \boldsymbol{x}, \quad\|\boldsymbol{v}\|_{\mathrm{V}_{1}(\Omega)}:=\left(\int_{\Omega}|\mathcal{E}(\boldsymbol{v})|^{2} \boldsymbol{d} \boldsymbol{x}\right)^{1 / 2} .
$$

The Korn inequality [13, Ch. I, Sect. 2.2]

$$
\|\boldsymbol{v}\|_{\mathbf{H}^{1}(\Omega)} \leqslant C\|\mathcal{E}(\boldsymbol{v})\|_{\mathbf{L}_{2}(\Omega)}, \quad C=\text { const }, \quad \forall \boldsymbol{v} \in \mathbf{H}_{0}^{1}(\Omega),
$$

playing a fundamental role in many problems of plasticity and elasticity, implies that the above introduced norm $\|\cdot\|_{\mathbf{V}_{1}(\Omega)}$ is equivalent to the norm $\|\cdot\|_{\mathbf{H}^{1}(\Omega)}$.

In the space $\mathbf{V}_{3}(\Omega)$ we introduce a scalar product and a norm by the following identities:

$$
\begin{gathered}
(\boldsymbol{v}, \boldsymbol{w})_{\mathbf{V}_{3}(\Omega)}:=\int_{\Omega} \nabla_{\mathbf{x}}^{3} \boldsymbol{v}: \nabla_{\mathrm{x}}^{3} \boldsymbol{w} \boldsymbol{d} \boldsymbol{x}+\int_{\Omega} \nabla_{\mathrm{x}}^{2} \boldsymbol{v} \cdot \nabla_{\mathrm{x}}^{2} \boldsymbol{w} \boldsymbol{d} \boldsymbol{x}, \\
\|\boldsymbol{v}\|_{\mathbf{V}_{3}(\Omega)}:=\left(\int_{\Omega}\left|\nabla_{\mathbf{x}}^{3} \boldsymbol{v}\right|^{2} \boldsymbol{d} \boldsymbol{x}+\int_{\Omega}\left|\nabla_{\mathbf{x}}^{2} \boldsymbol{v}\right|^{2} \boldsymbol{d} \boldsymbol{x}\right)^{1 / 2} .
\end{gathered}
$$

Lemma 2.1. Let $\partial \Omega \in \mathcal{C}^{3}$. Then the norms $\|\cdot\|_{\mathbf{V}_{3}(\Omega)}$ and $\|\cdot\|_{\mathbf{H}^{3}(\Omega)}$ are equivalent, that is, there exist positive constants $K_{1}$ and $K_{2}$ such that

$$
K_{1}\|\boldsymbol{v}\|_{\mathbf{H}^{3}(\Omega)} \leqslant\|\boldsymbol{v}\|_{\mathbf{V}_{3}(\Omega)} \leqslant K_{2}\|\boldsymbol{v}\|_{\mathbf{H}^{3}(\Omega)}, \quad \forall \boldsymbol{v} \in \mathbf{V}_{3}(\Omega) .
$$

Proof. The right inequality in (2.1) is obviously implied by the definition of the norm in the Sobolev space $\mathbf{H}^{3}(\Omega)$.

We note that by classical results on properties of solutions to stationary Stokes equations subject to the Dirichlet condition, see, for instance, [18, Ch. I, Sect. 2], the following estimate holds:

$$
\|\boldsymbol{v}\|_{\mathbf{H}^{3}(\Omega)} \leqslant \widetilde{K}_{1}\left\|\mathbf{P}_{\mathrm{L}}\left(\Delta_{\mathbf{x}} \boldsymbol{v}\right)\right\|_{\mathbf{H}^{1}(\Omega)}, \quad \forall \boldsymbol{v} \in \mathbf{V}_{3}(\Omega)
$$


where $\mathbf{P}_{\mathrm{L}}$ is the Leray projector, $\widetilde{K}_{1}$ is a positive constant.

Since

$$
\mathbf{P}_{\mathrm{L}}\left(\Delta_{\mathrm{x}} \phi\right)=\Delta_{\mathrm{x}} \phi
$$

for each vector function $\phi \in \mathcal{V}(\Omega)$, then inequality 2.2$)$ implies that

$$
\|\phi\|_{\mathbf{H}^{3}(\Omega)} \leqslant \widetilde{K}_{1}\left\|\Delta_{\mathbf{x}} \phi\right\|_{\mathbf{H}^{1}(\Omega)}, \quad \forall \phi \in \mathcal{V}(\Omega) .
$$

The latter inequality is extended by continuity on the closure $\mathcal{V}(\Omega)$ in $\mathbf{H}^{3}(\Omega)$ that leads us to the relation

$$
\|\boldsymbol{v}\|_{\mathbf{H}^{3}(\Omega)} \leqslant \widetilde{K}_{1}\left\|\Delta_{\mathbf{x}} \boldsymbol{v}\right\|_{\mathbf{H}^{1}(\Omega)}, \quad \forall \boldsymbol{v} \in \mathbf{V}_{3}(\Omega)
$$

This implies the left inequality in 2.1 with $K_{1}=1 / \widetilde{K}_{1}$. The proof is complete.

Let us describe conditions imposed for the data of model (1.3). We assume that

(i) the surface $\partial \Omega$ has the smoothness of $\operatorname{class} \mathcal{C}^{3}$;

(ii) the inclusion holds $\boldsymbol{f} \in \mathbf{H}^{-1}(\Omega)$;

(iii) the viscosity $\nu: \mathbb{R}_{+} \rightarrow \mathbb{R}_{+}$is continuous and there exist "limiting Newton viscosities" $\nu_{0}$ and $\nu_{1}$, that is, $0<\nu_{0} \leqslant \nu(\tau) \leqslant \nu_{1}$ for each $\tau \in \mathbb{R}_{+}$;

(iv) the operator $\mathcal{P} \mathcal{E}: \mathbf{V}_{1}(\Omega) \rightarrow \mathbf{L}_{2}(\Omega)$ is strongly continuous, that is, the weak convergence $\boldsymbol{u}_{k} \rightarrow \boldsymbol{u}_{0}$ in the space $\mathbf{V}_{1}(\Omega)$ implies the strong convergence $\mathcal{P} \mathcal{E}\left(\boldsymbol{u}_{k}\right) \rightarrow \mathcal{P} \mathcal{E}\left(\boldsymbol{u}_{0}\right)$ in $\mathbf{L}_{2}(\Omega)$ as $k \rightarrow \infty$.

Remark 2.1. As an example of the operator $\mathcal{P}$ obeying property (iv), we can take the averaging operator [19, Ch. I, Sect. 1]; the usage of this operator in hydrodynamical models goes back to works by O.A. Ladyzhenskaya.

Definition 2.1. A weak solution of boundary value problem (1.3), (1.4) is a pair $(\boldsymbol{v}, \pi) \in$ $\mathbf{V}_{1}(\Omega) \times \mathfrak{D}^{\prime}(\Omega)$ satisfying the first equation in $(1.3)$ in the sense of distributions, that is,

$$
\begin{aligned}
-\sum_{i=1}^{n} \int_{\Omega} v_{i} \boldsymbol{v} \cdot \frac{\partial \boldsymbol{\varphi}}{\partial x_{i}} \boldsymbol{d} \boldsymbol{x} & +\int_{\Omega} \nu(|\boldsymbol{\mathcal { E }}(\boldsymbol{v})|) \mathcal{E}(\boldsymbol{v}): \mathcal{E}(\boldsymbol{\varphi}) \boldsymbol{d} \boldsymbol{x}-\kappa \sum_{i=1}^{n} \int_{\Omega} v_{i} \mathcal{E}(\boldsymbol{v}): \frac{\partial \mathcal{E}(\boldsymbol{\varphi})}{\partial x_{i}} \boldsymbol{d x} \\
& -\left\langle\pi, \operatorname{div}_{\mathbf{x}} \boldsymbol{\varphi}\right\rangle_{\mathfrak{D}^{\prime}(\Omega) \times \mathfrak{D}(\Omega)}=\langle\boldsymbol{f}, \boldsymbol{\varphi}\rangle_{\mathbf{H}^{-1}(\Omega) \times \mathbf{H}_{0}^{1}(\Omega)}
\end{aligned}
$$

for each vector function $\varphi \in \mathfrak{D}(\Omega)$.

The main result of the present work is formulated as the following existence theorem.

Theorem 2.1. Under Conditions (i)-(iv), problem (1.3), (1.4) possesses at least one weak solution $(\boldsymbol{v}, \pi) \in \mathbf{V}_{1}(\Omega) \times \mathfrak{D}^{\prime}(\Omega)$ and

$$
\int_{\Omega} \nu(|\mathcal{P} \mathcal{E}(\boldsymbol{v})|)|\mathcal{E}(\boldsymbol{v})|^{2} \boldsymbol{d} \boldsymbol{x} \leqslant\langle\boldsymbol{f}, \boldsymbol{v}\rangle_{\mathbf{H}^{-1}(\Omega) \times \mathbf{H}_{0}^{1}(\Omega)} .
$$

The proof of this theorem is given in Section 5 .

\section{Solvability OF SOlutions With $\alpha$-OPERATORS}

Following [15, Ch. 2], we recall some preliminaries on $\alpha$-operators. The content of this section plays an important role in the proof of Theorem 2.1 .

Let $\mathbf{E}$ be a real reflexive separable Banach space with the norm $\|\cdot\|_{\mathbf{E}}$ and $\mathbf{E}^{*}$ be a dual space for $\mathbf{E}$. 
Definition 3.1. An operator $\mathbf{A}: \mathbf{E} \rightarrow \mathbf{E}^{*}$ satisfies $\alpha$-condition if for an arbitrary sequence $\left\{\mathbf{u}_{k}\right\}_{k=1}^{\infty} \subset \mathbf{E}$, the weak convergence $\mathbf{u}_{k} \rightarrow \mathbf{u}_{0}$ and the inequality

$$
\varlimsup_{k \rightarrow \infty}\left\langle\mathbf{A}\left(\mathbf{u}_{k}\right), \mathbf{u}_{k}-\mathbf{u}_{0}\right\rangle_{\mathbf{E}^{*} \times \mathbf{E}} \leqslant 0
$$

implies the strong convergence $\mathbf{u}_{k} \rightarrow \mathbf{u}_{0}$ in the norm of $\mathbf{E}$.

For the sake of brevity, an operator obeying $\alpha$-condition will be sometimes called $\alpha$-operator.

Lemma 3.1. If $\mathbf{A}: \mathbf{E} \rightarrow \mathbf{E}^{*}$ is an $\alpha$-operator, $\mathbf{K}: \mathbf{E} \rightarrow \mathbf{E}^{*}$ is a strongly continuous operator, then the sum $\mathbf{A}+\mathbf{K}$ satisfies $\alpha$-condition.

Lemma 3.2. If $\mathbf{Q}: \mathbf{E} \rightarrow \mathbf{E}^{*}$ is a strongly monotone operator, that is,

$$
\langle\mathbf{Q}(\mathbf{u})-\mathbf{Q}(\mathbf{v}), \mathbf{u}-\mathbf{v}\rangle_{\mathbf{E}^{*} \times \mathbf{E}} \geqslant C\|\mathbf{u}-\mathbf{v}\|_{\mathbf{E}}^{2}, \quad C=\text { const } \quad \forall \mathbf{u}, \mathbf{v} \in \mathbf{E},
$$

then $\mathbf{Q}$ is an $\alpha$-operator.

Theorem 3.1 (Acute angle theorem). Let $\mathbf{D}$ be an open bounded subset $\mathbf{E}$ and $\mathbf{0} \in \mathbf{D}$. If the mapping $\mathbf{A}: \overline{\mathbf{D}} \subset \mathbf{E} \rightarrow \mathbf{E}^{*}$ is demi-continuous and bounded, satisfies $\alpha$-condition and the inequality

$$
\langle\mathbf{A}(\mathbf{u})-\mathbf{h}, \mathbf{u}\rangle_{\mathbf{E}^{*} \times \mathbf{E}}>0, \quad \forall \mathbf{u} \in \partial \mathbf{D},
$$

holds, then the equation $\mathbf{A}(\mathbf{u})=\mathbf{h}$ has at least one solution $\mathbf{u}_{\mathbf{h}} \in \mathbf{D}$.

\section{Solvability of FAMily of REgUlarized PROBlems}

Following the lines of [4], see also [6], we consider a one-parameter family of regularized problem: for a given $m \in \mathbb{N}$, we need to find a vector function $\boldsymbol{v}_{m} \in \mathbf{V}_{3}(\Omega)$ such that

$$
\begin{aligned}
\frac{1}{m} \int_{\Omega} \nabla_{\mathbf{x}}^{3} \boldsymbol{v}_{m}: \nabla_{\mathbf{x}}^{3} \boldsymbol{w} \boldsymbol{d} \boldsymbol{x} & +\frac{1}{m} \int_{\Omega} \nabla_{\mathbf{x}}^{2} \boldsymbol{v}_{m} \cdot \nabla_{\mathbf{x}}^{2} \boldsymbol{w} \boldsymbol{d} \boldsymbol{x}-\sum_{i=1}^{n} \int_{\Omega} v_{m i} \boldsymbol{v}_{m} \cdot \frac{\partial \boldsymbol{w}}{\partial x_{i}} \boldsymbol{d} \boldsymbol{x} \\
& +\int_{\Omega} \nu\left(\left|\mathcal{P} \mathcal{E}\left(\boldsymbol{v}_{m}\right)\right|\right) \mathcal{E}\left(\boldsymbol{v}_{m}\right): \mathcal{E}(\boldsymbol{w}) \boldsymbol{d} \boldsymbol{x} \\
& -\kappa \sum_{i=1}^{n} \int_{\Omega} v_{m i} \mathcal{E}\left(\boldsymbol{v}_{m}\right): \frac{\partial \mathcal{E}(\boldsymbol{w})}{\partial x_{i}} \boldsymbol{d} \boldsymbol{x}=\langle\boldsymbol{f}, \boldsymbol{w}\rangle_{\mathbf{H}^{-1}(\Omega) \times \mathbf{H}_{0}^{1}(\Omega)}
\end{aligned}
$$

for each $\boldsymbol{w} \in \mathbf{V}_{3}(\Omega)$.

Lemma 4.1. Under Conditions (i)-(iv), problem (4.1) has at least one solution $\boldsymbol{v}_{m} \in \mathbf{V}_{3}(\Omega)$ and the inequality

$$
\frac{1}{m}\left\|\boldsymbol{v}_{m}\right\|_{\mathbf{V}_{3}(\Omega)}^{2}+\nu_{0}\left\|\boldsymbol{v}_{m}\right\|_{\mathbf{V}_{1}(\Omega)}^{2} \leqslant\left\langle\boldsymbol{f}, \boldsymbol{v}_{m}\right\rangle_{\mathbf{H}^{-1}(\Omega) \times \mathbf{H}_{0}^{1}(\Omega)}
$$

holds. 
Proof. We introduce operators $\mathbf{A}_{m}, \mathbf{B}_{1}, \mathbf{B}_{2}, \mathbf{B}_{3}, \mathbf{B}$ and $\mathbf{J}$ :

$$
\begin{aligned}
& \mathbf{A}_{m}: \mathbf{V}_{3}(\Omega) \rightarrow \mathbf{V}_{3}^{*}(\Omega), \\
& \left\langle\mathbf{A}_{m}(\boldsymbol{v}), \boldsymbol{w}\right\rangle_{\mathbf{V}_{3}^{*}(\Omega) \times \mathbf{V}_{3}(\Omega)}:=\frac{1}{m} \int_{\Omega} \nabla_{\mathbf{x}}^{3} \boldsymbol{v}: \nabla_{\mathbf{x}}^{3} \boldsymbol{w} \boldsymbol{d} \boldsymbol{x}+\frac{1}{m} \int_{\Omega} \nabla_{\mathbf{x}}^{2} \boldsymbol{v} \cdot \nabla_{\mathbf{x}}^{2} \boldsymbol{w} \boldsymbol{d} \boldsymbol{x}, \\
& \mathbf{B}_{1}: \mathbf{V}_{2}(\Omega) \rightarrow \mathbf{V}_{3}^{*}(\Omega), \quad\left\langle\mathbf{B}_{1}(\boldsymbol{v}), \boldsymbol{w}\right\rangle_{\mathbf{V}_{3}^{*}(\Omega) \times \mathbf{V}_{3}(\Omega)}:=-\sum_{i=1}^{n} \int_{\Omega} v_{i} \boldsymbol{v} \cdot \frac{\partial \boldsymbol{w}}{\partial x_{i}} \boldsymbol{d} \boldsymbol{x}, \\
& \mathbf{B}_{2}: \mathbf{V}_{2}(\Omega) \rightarrow \mathbf{V}_{3}^{*}(\Omega), \quad\left\langle\mathbf{B}_{2}(\boldsymbol{v}), \boldsymbol{w}\right\rangle_{\mathbf{V}_{3}^{*}(\Omega) \times \mathbf{V}_{3}(\Omega)}:=\int_{\Omega} \nu(|\mathcal{P} \mathcal{E}(\boldsymbol{v})|) \mathcal{E}(\boldsymbol{v}): \mathcal{E}(\boldsymbol{w}) \boldsymbol{d} \boldsymbol{x}, \\
& \mathbf{B}_{3}: \mathbf{V}_{2}(\Omega) \rightarrow \mathbf{V}_{3}^{*}(\Omega), \quad\left\langle\mathbf{B}_{3}(\boldsymbol{v}), \boldsymbol{w}\right\rangle_{\mathbf{V}_{3}^{*}(\Omega) \times \mathbf{V}_{3}(\Omega)}:=-\kappa \sum_{i=1}^{n} \int_{\Omega} v_{i} \mathcal{E}(\boldsymbol{v}): \frac{\partial \mathcal{E}(\boldsymbol{w})}{\partial x_{i}} \boldsymbol{d} \boldsymbol{x}, \\
& \mathbf{B}: \mathbf{V}_{2}(\Omega) \rightarrow \mathbf{V}_{3}^{*}(\Omega), \quad \mathbf{B}:=\mathbf{B}_{1}+\mathbf{B}_{2}+\mathbf{B}_{3}, \\
& \mathbf{J}: \mathbf{V}_{3}(\Omega) \rightarrow \mathbf{V}_{2}(\Omega), \quad \mathbf{J}(\boldsymbol{v}):=\boldsymbol{v}
\end{aligned}
$$

and rewrite problem (4.1) in an equivalent form:

$$
\mathbf{A}_{m}\left(\boldsymbol{v}_{m}\right)+\mathbf{B} \circ \mathbf{J}\left(\boldsymbol{v}_{m}\right)=\boldsymbol{f}
$$

It is clear that the operator $\mathbf{A}_{m}$ is continuous and the identities holds:

$$
\begin{aligned}
\left\langle\mathbf{A}_{m}(\boldsymbol{v})-\mathbf{A}_{m}(\boldsymbol{u}), \boldsymbol{v}-\boldsymbol{u}\right\rangle & =\frac{1}{m} \int_{\Omega}\left|\nabla_{\mathbf{x}}^{3}(\boldsymbol{v}-\boldsymbol{u})\right|^{2} \boldsymbol{d} \boldsymbol{x}+\frac{1}{m} \int_{\Omega}\left|\nabla_{\mathbf{x}}^{2}(\boldsymbol{v}-\boldsymbol{u})\right|^{2} \boldsymbol{d} \boldsymbol{x} \\
& =\frac{1}{m}\|\boldsymbol{v}-\boldsymbol{u}\|_{\mathbf{V}_{3}(\Omega)}^{2} .
\end{aligned}
$$

Then, according to Lemma 3.2 , this operator satisfies $\alpha$-condition.

Since the embedding $H^{3}(\Omega) \subset H^{2}(\Omega)$ is compact, the same is true for the embedding $\mathbf{V}_{3}(\Omega) \subset \mathbf{V}_{2}(\Omega)$. This is why the operator $\mathbf{J}$ is strongly continuous.

We are going to show that the operator $\mathbf{B}$ is continuous. First we consider the first term $\mathbf{B}_{1}$. We choose a sequence of vector functions $\left\{\boldsymbol{u}_{\ell}\right\}_{\ell=1}^{\infty}$ such that $\boldsymbol{u}_{\ell} \rightarrow \boldsymbol{u}_{0}$ strongly in $\mathbf{V}_{2}(\Omega)$ as $\ell \rightarrow \infty$. In particular this implies that $\boldsymbol{u}_{\ell} \rightarrow \boldsymbol{u}_{0}$ in the norm of the space $\mathbf{L}_{4}(\Omega)$, see [17, Ch. 6]. We need to establish that $\mathbf{B}_{1}\left(\boldsymbol{u}_{\ell}\right) \rightarrow \mathbf{B}_{1}\left(\boldsymbol{u}_{0}\right)$ strongly in $\mathbf{V}_{3}^{*}(\Omega)$ as $\ell \rightarrow \infty$. Let $\boldsymbol{w}$ be an arbitrary vector function in the space $\mathbf{V}_{3}(\Omega)$. Since

$$
\begin{aligned}
\left\langle\mathbf{B}_{1}\left(\boldsymbol{u}_{\ell}\right)-\mathbf{B}_{1}\left(\boldsymbol{u}_{0}\right), \boldsymbol{w}\right\rangle_{\mathbf{V}_{3}^{*}(\Omega) \times \mathbf{V}_{3}(\Omega)}= & -\sum_{i=1}^{n} \int_{\Omega} u_{\ell i} \boldsymbol{u}_{\ell} \cdot \frac{\partial \boldsymbol{w}}{\partial x_{i}} \boldsymbol{d} \boldsymbol{x}+\sum_{i=1}^{n} \int_{\Omega} u_{0 i} \boldsymbol{u}_{0} \cdot \frac{\partial \boldsymbol{w}}{\partial x_{i}} \boldsymbol{d} \boldsymbol{x} \\
= & \sum_{i=1}^{n} \int_{\Omega}\left(u_{0 i}-u_{\ell i}\right) \boldsymbol{u}_{\ell} \cdot \frac{\partial \boldsymbol{w}}{\partial x_{i}} \boldsymbol{d} \boldsymbol{x} \\
& +\sum_{i=1}^{n} \int_{\Omega} u_{0 i}\left(\boldsymbol{u}_{0}-\boldsymbol{u}_{\ell}\right) \cdot \frac{\partial \boldsymbol{w}}{\partial x_{i}} \boldsymbol{d x},
\end{aligned}
$$

by Hölder inequality we get the following estimate:

$\left|\left\langle\mathbf{B}_{1}\left(\boldsymbol{u}_{\ell}\right)-\mathbf{B}_{1}\left(\boldsymbol{u}_{0}\right), \boldsymbol{w}\right\rangle_{\mathbf{V}_{3}^{*}(\Omega) \times \mathbf{V}_{3}(\Omega)}\right| \leqslant C\left\|\boldsymbol{u}_{\ell}-\boldsymbol{u}_{0}\right\|_{\mathbf{L}_{4}}(\Omega) \max \left\{\left\|\boldsymbol{u}_{k}\right\|_{\mathbf{L}_{4}}(\Omega): k=0,1,2, \ldots\right\}\|\boldsymbol{w}\|_{\mathbf{V}_{3}(\Omega)}$, with some constant $C$ independent of the index $\ell$. Therefore,

$$
\left\|\mathbf{B}_{1}\left(\boldsymbol{u}_{\ell}\right)-\mathbf{B}_{1}\left(\boldsymbol{u}_{0}\right)\right\|_{\mathbf{V}_{3}^{*}(\Omega)} \leqslant C\left\|\boldsymbol{u}_{\ell}-\boldsymbol{u}_{0}\right\|_{\mathbf{L}_{4}(\Omega)} \max \left\{\left\|\boldsymbol{u}_{k}\right\|_{\mathbf{L}_{4}(\Omega)}: k=0,1,2, \ldots\right\} \rightarrow 0
$$

as $\ell \rightarrow \infty$, that is, we have the strong convergence $\mathbf{B}_{1}\left(\boldsymbol{u}_{\ell}\right) \rightarrow \mathbf{B}_{1}\left(\boldsymbol{u}_{0}\right)$ in $\mathbf{V}_{3}^{*}(\Omega)$. 
By similar arguing one can establish the continuity of the operators $\mathbf{B}_{2}$ and $\mathbf{B}_{3}$.

Thus, the sum of the operators $\mathbf{B}=\mathbf{B}_{1}+\mathbf{B}_{2}+\mathbf{B}_{3}$ is continuous and the composition $\mathbf{B} \circ \mathbf{J}$ is strongly continuous. Applying Lemma 3.1, we conclude that the mapping $\mathbf{A}_{m}+\mathbf{B} \circ \mathbf{J}$ satisfies $\alpha$-condition.

We note that

$$
\left\langle\mathbf{A}_{m}(\boldsymbol{v}), \boldsymbol{v}\right\rangle_{\mathbf{V}_{3}^{*}(\Omega) \times \mathbf{V}_{3}(\Omega)}=\frac{1}{m}\|\boldsymbol{v}\|_{\mathbf{V}_{3}(\Omega)}^{2} .
$$

Integrating by parts, we also obtain that

$$
\begin{aligned}
\sum_{i=1}^{n} \int_{\Omega} v_{i} \boldsymbol{v} \cdot \frac{\partial \boldsymbol{v}}{\partial x_{i}} \boldsymbol{d} \boldsymbol{x} & =\frac{1}{2} \sum_{i=1}^{n} \int_{\Omega} v_{i} \frac{\partial|\boldsymbol{v}|^{2}}{\partial x_{i}} \boldsymbol{d} \boldsymbol{x} \\
& =\frac{1}{2} \int_{\partial \Omega}(\boldsymbol{v} \cdot \mathbf{n})|\boldsymbol{v}|^{2} \boldsymbol{d} \boldsymbol{x}-\frac{1}{2} \int_{\Omega}\left(\operatorname{div}_{\mathbf{x}} \boldsymbol{v}\right)|\boldsymbol{v}|^{2} \boldsymbol{d} \boldsymbol{x}=0 \\
\sum_{i=1}^{n} \int_{\Omega} v_{i} \mathcal{E}(\boldsymbol{v}): \frac{\partial \mathcal{E}(\boldsymbol{v})}{\partial x_{i}} \boldsymbol{d} \boldsymbol{x} & =\frac{1}{2} \sum_{i=1}^{n} \int_{\Omega} v_{i} \frac{\partial|\mathcal{E}(\boldsymbol{v})|^{2}}{\partial x_{i}} \boldsymbol{d x} \\
& =\frac{1}{2} \int_{\partial \Omega}(\boldsymbol{v} \cdot \mathbf{n})|\mathcal{E}(\boldsymbol{v})|^{2} \boldsymbol{d} \boldsymbol{x}-\frac{1}{2} \int_{\Omega}\left(\operatorname{div}_{\mathbf{x}} \boldsymbol{v}\right)|\mathcal{E}(\boldsymbol{v})|^{2} \boldsymbol{d} \boldsymbol{x}=0,
\end{aligned}
$$

where the symbol $\mathbf{n}$ denotes the outward normal to the surface $\partial \Omega$. This is why by Condition (iii) we get the estimate:

$$
\langle\mathbf{B} \circ \mathbf{J}(\boldsymbol{v}), \boldsymbol{v}\rangle_{\mathbf{V}_{3}^{*}(\Omega) \times \mathbf{V}_{3}(\Omega)} \geqslant \nu_{0}\|\boldsymbol{v}\|_{\mathbf{V}_{1}(\Omega)}^{2} .
$$

It follows from relations 4.4 and 4.7 that

$$
\left\langle\mathbf{A}_{m}(\boldsymbol{v})+\mathbf{B} \circ \mathbf{J}(\boldsymbol{v}), \boldsymbol{v}\right\rangle_{\mathbf{V}_{3}^{*}(\Omega) \times \mathbf{V}_{3}(\Omega)} \geqslant \frac{1}{m}\|\boldsymbol{v}\|_{\mathbf{V}_{3}(\Omega)}^{2} .
$$

This easily implies the estimate

$$
\begin{aligned}
\left\langle\mathbf{A}_{m}(\boldsymbol{v})+\mathbf{B} \circ \mathbf{J}(\boldsymbol{v})-\boldsymbol{f}, \boldsymbol{v}\right\rangle_{\mathbf{V}_{3}^{*}(\Omega) \times \mathbf{V}_{3}(\Omega)} & \geqslant \frac{1}{m}\|\boldsymbol{v}\|_{\mathbf{V}_{3}(\Omega)}^{2}-\langle\boldsymbol{f}, \boldsymbol{v}\rangle_{\mathbf{V}_{3}^{*}(\Omega) \times \mathbf{V}_{3}(\Omega)} \\
& \geqslant\left(\frac{1}{m}\|\boldsymbol{v}\|_{\mathbf{V}_{3}(\Omega)}-\|\boldsymbol{f}\|_{\mathbf{V}_{3}^{*}(\Omega)}\right)\|\boldsymbol{v}\|_{\mathbf{V}_{3}(\Omega)}>0
\end{aligned}
$$

under the condition that

$$
\|\boldsymbol{v}\|_{\mathbf{v}_{3}(\Omega)}>m\|\boldsymbol{f}\|_{\mathbf{V}_{3}^{*}(\Omega)} .
$$

Applying Theorem 3.1 to 4.3), we confirm that problem 4.1) has a solution $\boldsymbol{v}_{m}$ in the ball $\mathfrak{B}_{R} \subset \mathbf{V}_{3}(\Omega)$ of radius $R$ for each $R>m\|\boldsymbol{f}\|_{\mathbf{V}_{3}^{*}(\Omega)}$ and centered as $\mathbf{0}$. Estimate (4.2) is obtained by substituting $\boldsymbol{w}=\boldsymbol{v}_{m}$ into (4.1) and taking into consideration identities 4.5) and (4.6) with $\boldsymbol{v}=\boldsymbol{v}_{m}$. The proof is complete.

\section{Proof of Theorem 2.1}

We consider a sequence $\left\{\boldsymbol{v}_{m}\right\}_{m=1}^{\infty}$ of solutions of problems 4.1). It follows from estimate 4.2 that

$$
\nu_{0}\left\|\boldsymbol{v}_{m}\right\|_{\mathbf{V}_{1}(\Omega)}^{2} \leqslant\|\boldsymbol{f}\|_{\mathbf{H}^{-1}(\Omega)}\left\|\boldsymbol{v}_{m}\right\|_{\mathbf{H}_{0}^{1}(\Omega)} \leqslant C\|\boldsymbol{f}\|_{\mathbf{H}^{-1}(\Omega)}\left\|\boldsymbol{v}_{m}\right\|_{\mathbf{V}_{1}(\Omega)}, \quad C=\text { const }
$$

and hence,

$$
\left\|\boldsymbol{v}_{m}\right\|_{\mathbf{v}_{1}(\Omega)} \leqslant C \nu_{0}^{-1}\|\boldsymbol{f}\|_{\mathbf{H}^{-1}(\Omega)} .
$$

This is why, without loss of generality, we can assume that

$$
\begin{aligned}
& \boldsymbol{v}_{m} \rightarrow \boldsymbol{v} \quad \text { weakly in } \mathbf{V}_{1}(\Omega) \text { as } m \rightarrow \infty, \\
& \boldsymbol{v}_{m} \rightarrow \boldsymbol{v} \text { in the norm of } \mathbf{L}_{2}(\Omega) \text { as } m \rightarrow \infty
\end{aligned}
$$


for some vector function $\boldsymbol{v}$ in the space $\mathbf{V}_{1}(\Omega)$. Here we have employed the compactness of the embedding $\mathbf{H}^{1}(\Omega) \subset \mathbf{L}_{2}(\Omega)$, see [17, Ch. 6].

In view of Condition (iv), we obtain:

$$
\mathcal{P E}\left(\boldsymbol{v}_{m}\right) \rightarrow \mathcal{P} \mathcal{E}(\boldsymbol{v}) \text { in the norm of } \mathbf{L}_{2}(\Omega) \text { as } m \rightarrow \infty,
$$

which implies that

$$
\mathcal{P E}\left(\boldsymbol{v}_{m_{k}}\right) \rightarrow \mathcal{P} \mathcal{E}(\boldsymbol{v}) \quad \text { a.e. in } \Omega \text { as } k \rightarrow \infty
$$

for some subsequence $\left\{m_{k}\right\}_{k=1}^{\infty}$. Without loss of generality we assume that

$$
\mathcal{P} \mathcal{E}\left(\boldsymbol{v}_{m}\right) \rightarrow \mathcal{P} \mathcal{E}(\boldsymbol{v}) \text { a.e. in } \Omega \text { as } m \rightarrow \infty .
$$

Since the function $\nu$ is continuous, see Condition (iii), we have:

$$
\nu\left(\left|\mathcal{P} \mathcal{E}\left(\boldsymbol{v}_{m}\right)\right|\right) \rightarrow \nu(|\mathcal{P} \mathcal{E}(\boldsymbol{v})|) \text { a.e. in } \Omega \text { as } m \rightarrow \infty
$$

and therefore,

$$
\left|\nu\left(\left|\mathcal{P} \mathcal{E}\left(\boldsymbol{v}_{m}\right)\right|\right)-\nu(|\mathcal{P} \mathcal{E}(\boldsymbol{v})|)\right|^{2} \rightarrow 0 \quad \text { a.e. in } \Omega \text { as } m \rightarrow \infty \text {. }
$$

We observe that due to Condition (iii), the function $\nu$ is bounded. This is why we can apply the Lebesgue theorem on dominated convergence to the sequence of functions $\left\{\mid \nu\left(\left|\mathcal{P} \mathcal{E}\left(\boldsymbol{v}_{m}\right)\right|\right)-\right.$ $\left.\left.\nu(|\mathcal{P} \mathcal{E}(\boldsymbol{v})|)\right|^{2}\right\}_{m=1}^{\infty}$. We then obtain that point-wise convergence $(5.4)$ implies that

$$
\nu\left(\left|\mathcal{P} \mathcal{E}\left(\boldsymbol{v}_{m}\right)\right|\right) \rightarrow \nu(|\mathcal{P} \mathcal{E}(\boldsymbol{v})|) \quad \text { in the norm } L_{2}(\Omega) \text { as } m \rightarrow \infty .
$$

Now we choose an arbitrary vector function $\boldsymbol{\psi} \in \mathcal{V}(\Omega)$. Since $\boldsymbol{v}_{m}$ is a solution of (4.1), we see that

$$
\begin{aligned}
\frac{1}{m} \int_{\Omega} \nabla_{\mathbf{x}}^{3} \boldsymbol{v}_{m}: \nabla_{\mathbf{x}}^{3} \boldsymbol{\psi} \boldsymbol{d} \boldsymbol{x} & +\frac{1}{m} \int_{\Omega} \nabla_{\mathbf{x}}^{2} \boldsymbol{v}_{m} \cdot \nabla_{\mathbf{x}}^{2} \boldsymbol{\psi} \boldsymbol{d} \boldsymbol{x}-\sum_{i=1}^{n} \int_{\Omega} v_{m i} \boldsymbol{v}_{m} \cdot \frac{\partial \boldsymbol{\psi}}{\partial x_{i}} \boldsymbol{d} \boldsymbol{x} \\
& +\int_{\Omega} \nu\left(\left|\mathcal{P} \mathcal{E}\left(\boldsymbol{v}_{m}\right)\right|\right) \mathcal{E}\left(\boldsymbol{v}_{m}\right): \mathcal{E}(\boldsymbol{\psi}) \boldsymbol{d} \boldsymbol{x} \\
& -\kappa \sum_{i=1}^{n} \int_{\Omega} v_{m i} \mathcal{E}\left(\boldsymbol{v}_{m}\right): \frac{\partial \mathcal{E}(\boldsymbol{\psi})}{\partial x_{i}} \boldsymbol{d} \boldsymbol{x}=\langle\boldsymbol{f}, \boldsymbol{\psi}\rangle_{\mathbf{H}^{-1}(\Omega) \times \mathbf{H}_{0}^{1}(\Omega)} .
\end{aligned}
$$

After appropriated number of integration by parts in the first and second terms in the left hand side of the above identity we find:

$$
\begin{aligned}
-\frac{1}{m} \int_{\Omega} \boldsymbol{v}_{m} \cdot \nabla_{\mathbf{x}}^{6} \boldsymbol{\psi} \boldsymbol{d} \boldsymbol{x} & +\frac{1}{m} \int_{\Omega} \boldsymbol{v}_{m} \cdot \nabla_{\mathbf{x}}^{4} \boldsymbol{\psi} \boldsymbol{d} \boldsymbol{x}-\sum_{i=1}^{n} \int_{\Omega} v_{m i} \boldsymbol{v}_{m} \cdot \frac{\partial \boldsymbol{\psi}}{\partial x_{i}} \boldsymbol{d} \boldsymbol{x} \\
& +\int_{\Omega} \nu\left(\left|\mathcal{P} \mathcal{E}\left(\boldsymbol{v}_{m}\right)\right|\right) \mathcal{E}\left(\boldsymbol{v}_{m}\right): \mathcal{E}(\boldsymbol{\psi}) \boldsymbol{d} \boldsymbol{x} \\
& -\kappa \sum_{i=1}^{n} \int_{\Omega} v_{m i} \mathcal{E}\left(\boldsymbol{v}_{m}\right): \frac{\partial \mathcal{E}(\boldsymbol{\psi})}{\partial x_{i}} \boldsymbol{d} \boldsymbol{x}=\langle\boldsymbol{f}, \boldsymbol{\psi}\rangle_{\mathbf{H}^{-1}(\Omega) \times \mathbf{H}_{0}^{1}(\Omega)} .
\end{aligned}
$$

Employing (5.1), (5.2) and (5.5), we pass to the limit as $m \rightarrow \infty$ in identity (5.6):

$$
\begin{aligned}
-\sum_{i=1}^{n} \int_{\Omega} v_{i} \boldsymbol{v} \cdot \frac{\partial \boldsymbol{\psi}}{\partial x_{i}} \boldsymbol{d} \boldsymbol{x} & +\int_{\Omega} \nu(|\mathcal{P} \mathcal{E}(\boldsymbol{v})|) \mathcal{E}(\boldsymbol{v}): \mathcal{E}(\boldsymbol{\psi}) \boldsymbol{d} \boldsymbol{x} \\
& -\kappa \sum_{i=1}^{n} \int_{\Omega} v_{i} \mathcal{E}(\boldsymbol{v}): \frac{\partial \mathcal{E}(\boldsymbol{\psi})}{\partial x_{i}} \boldsymbol{d} \boldsymbol{x}=\langle\boldsymbol{f}, \boldsymbol{\psi}\rangle_{\mathbf{H}^{-1}(\Omega) \times \mathbf{H}_{0}^{1}(\Omega)}
\end{aligned}
$$


By De Rham theorem, see [18, Ch. I, Sect. 1.4], identity (5.7) implies that there exists $\pi \in \mathfrak{D}^{\prime}(\Omega)$ such that the first equation in system $(1.3)$ holds in the distribution sense. Hence, we have established that the pair $(\boldsymbol{v}, \pi)$ is a weak solution of boundary value problem (1.3), (1.4).

We substitute $\boldsymbol{w}=\boldsymbol{v}_{m}$ into (4.1). Since the third and fifth terms in the left hand side are zero, we readily see that

$$
\int_{\Omega} \nu\left(\left|\mathcal{P} \mathcal{E}\left(\boldsymbol{v}_{m}\right)\right|\right)\left|\mathcal{E}\left(\boldsymbol{v}_{m}\right)\right|^{2} \boldsymbol{d} \boldsymbol{x} \leqslant\left\langle\boldsymbol{f}, \boldsymbol{v}_{m}\right\rangle_{\mathbf{H}^{-1}(\Omega) \times \mathbf{H}_{0}^{1}(\Omega)} .
$$

Passing to the limit inferior as $m \rightarrow \infty$, we find that

$$
\varliminf_{m \rightarrow \infty} \int_{\Omega} \nu\left(\left|\mathcal{P} \mathcal{E}\left(\boldsymbol{v}_{m}\right)\right|\right)\left|\mathcal{E}\left(\boldsymbol{v}_{m}\right)\right|^{2} \boldsymbol{d} \boldsymbol{x} \leqslant\langle\boldsymbol{f}, \boldsymbol{v}\rangle_{\mathbf{H}^{-1}(\Omega) \times \mathbf{H}_{0}^{1}(\Omega)} .
$$

Now we consider a sequence of matrix-valued functions

$$
\left\{\sqrt{\nu\left(\left|\mathcal{P} \mathcal{E}\left(\boldsymbol{v}_{m}\right)\right|\right)} \mathcal{E}\left(\boldsymbol{v}_{m}\right)\right\}_{m=1}^{\infty}
$$

It follows from (5.1) and $(5.3)$ that

$$
\sqrt{\nu\left(\left|\mathcal{P} \mathcal{E}\left(\boldsymbol{v}_{m}\right)\right|\right)} \mathcal{E}\left(\boldsymbol{v}_{m}\right) \rightarrow \sqrt{\nu(|\mathcal{P} \mathcal{E}(\boldsymbol{v})|)} \mathcal{E}(\boldsymbol{v}) \quad \text { weakly in } \mathbf{L}_{2}(\Omega) \text { as } m \rightarrow \infty
$$

This is why the inequality holds:

$$
\int_{\Omega} \nu(|\mathcal{P} \mathcal{E}(\boldsymbol{v})|)|\mathcal{E}(\boldsymbol{v})|^{2} \boldsymbol{d} \boldsymbol{x} \leqslant \underline{\lim _{m \rightarrow \infty}} \int_{\Omega} \nu\left(\left|\mathcal{P} \mathcal{E}\left(\boldsymbol{v}_{m}\right)\right|\right)\left|\mathcal{E}\left(\boldsymbol{v}_{m}\right)\right|^{2} \boldsymbol{d} \boldsymbol{x},
$$

and together with (5.8), this obviously leads us to estimate (2.3). The proof of Theorem 2.1 is complete.

\section{BIBLIOGRAPHY}

1. V.A. Pavlovsky. On theoretical description of weak aqueous solutions of polymers // Dokl. AN SSSR. 200:4. 809-812 (1971). (in Russian).

2. A.P. Oskolkov. On the global solvability of a boundary value problem for a system of third order occuring in studying of motion of viscous fluid // Zap. Nauchn. Sem. LOMI. 27. 145-160 (1972). (in Russian).

3. A.P. Oskolkov. Uniqueness and global solvability for boundary-value problems for the equations of motion of water solutions of polymers // Zap. Nauchn. Sem. LOMI. 38. 98-136 (1973). (in Russian).

4. A.P. Oskolkov. Unsteady flows of viscoelastic fluids // Trudy MIAN SSSR. 159. 103-131 (1983). [Proc. Steklov Inst. Math., 159, 105-134 (1984).]

5. G.A. Sviridyuk. On a model of the dynamics of a weakly compressible viscoelastic fluid // Izv. VUZov. Matem. 1. 62-70 (1994). [Russian Math. (Iz. VUZ). 38:1, 59-68 (1994).]

6. A.V. Zvyagin. Investigation of the solvability of a stationary boundary problem for the mathematical model of a low concentrated aqueous polymer solutions // Vestn. Voronezh. Gos. Univ., Ser. Fiz. Mat. 1. 147-156 (2011). (in Russian).

7. E.S. Baranovskii. Global solutions for a model of polymeric flows with wall slip // Math. Meth. Appl. Sci. 40:14. 5035-5043 (2017).

8. O.A. Ladyzhenskaya. On the global unique solvability of some two-dimensional problems for the water solutions of polymers // J. Math. Sci. 99:1. 888-897 (2000).

9. E.S. Baranovskii. Flows of a polymer fluid in domain with impermeable boundaries // Comput. Math. Math. Phys. 54:10. 1589-1596 (2014). 
10. M.A. Artemov, E.S. Baranovskii. Boundary value problems for motion equations of polymeric fluids with nonlinear slip condition on solid walls // Trudy Inst. Mat. Mekh. UrO RAN. 21:1, 14-24 (2015).

11. E.S. Baranovskii. Mixed initial-boundary value problem for equations of motion of Kelvin-Voigt fluids // Comput. Math. Math. Phys. 56:7. 1363-1371 (2016).

12. M.A. Artemov, E.S. Baranovskii. Solvability of the Boussinesq approximation for water polymer solutions // Mathematics. 7:7, id 611 (2019).

13. V.G. Litvinov. Motion of a nonlinear-viscous fluid. Nauka, Moscow (1982). (in Russian).

14. A.C. Eringen. Nonlocal continuum field theories. Springer Verlag, New York (2002).

15. I.V. Skrypnik. Methods for analysis of nonlinear elliptic boundary value problems. Nauka, Moscow (1991). [Amer. Math. Soc., Providence, RI (1994).]

16. F. Carapau. One-dimensional viscoelastic fluid model where viscosity and normal stress coefficients depend on the shear rate // Nonl. Anal. Real World Appl. 11:5. 4342-4354 (2010).

17. R.A. Adams, J.J.F. Fournier. Sobolev spaces. Elsevier, Amsterdam (2003).

18. R. Temam. Navier-Stokes equations. Theory and numerical analysis. North-Holland Publ. Co., Amsterdam (1979).

19. O.A. Ladyzhenskaya. The mathematical theory of viscous incompressible flow. Nauka, Moscow (1970). [Gordon and Breach Sci. Publ., New York (1969).]

Mikhail Anatolievich Artemov,

Voronezh State University,

Universitetskaya sq. 1,

394018, Voronezh, Russia

E-mail: artemov_m_a@mail.ru

Yulia Nikolaevna Babkina, Voronezh State University, Universitetskaya sq. 1, 394018, Voronezh, Russia

E-mail: poiais.vsu@gmail.com 